\title{
Photo-Induced Fractionation of Water Isotopomers in the Martian Atmosphere
}

\author{
Bing-Ming Cheng, Eh Piew Chew and Chin-Ping Liu \\ Synchrotron Radiation Research Center, Hsinchu, Taiwan
}

Mohammed Bahou and Yuan-Pern Lee
Department of Chemistry, National Tsing Hua University, Hsinchu, Taiwan

Yuk L. Yung and M. F. Gerstell

Division of Geological and Planetary Sciences, California Institute of Technology, Pasadena

\begin{abstract}
The history and size of the water reservoirs on early Mars can be constrained using isotopic ratios of deuterium to hydrogen. We present new laboratory measurements of the ultraviolet cross-sections of $\mathrm{H}_{2} \mathrm{O}$ and its isotopomers, and modeling calculations in support of a photo-induced fractionation effect (PHIFE), that reconciles a discrepancy between past theoretical modeling and recent observations. This supports the hypothesis that Mars had an early warm atmosphere and has lost at least a 50-m global layer of water. Likely applications of PHIFE to other planetary atmospheres are sketched.
\end{abstract}

\section{Paradox and Hypothesis}

Circumstantial evidence strongly suggests a warmer past climate on Mars that permitted liquid water near the surface [Carr, 1996; Baker et al., 1992]. This is very different from the current cold, arid conditions. Among the few quantitative clues to the evolution of the Martian atmosphere are the isotopic signatures left by the processes that have modified the atmosphere over time. Most of the loss processes preferentially remove the lighter species. If we understand the fractionation caused by these processes, and know the current isotopic ratios of the various reservoirs, we can constrain the early atmosphere and its evolution to the present [McElroy, 1972; McElroy and Yung, 1976; Jakosky, 1993; Thiemens et al., 1995; Farquhar et al., 1998; Krasnopolsky et al., 1997].

Owen et al. [1988] discovered that Martian water is enriched in deuterium. The $\mathrm{D} / \mathrm{H}$ ratio deduced from their measurement is 6 times the terrestrial value. This constrains the amount of water that has escaped from the planet. But the result depends on a model-derived fractionation factor, $F$. The time dependence of the

Copyright 1999 by the American Geophysical Union.

Paper number 1999GL008367.

0094-8276/99/1999GL008367\$05.00
$\mathrm{D} / \mathrm{H}$ ratio may be written

$$
X(t)=X(0)[(W+L) / W]^{1-F}
$$

where $X(t)$ is the $\mathrm{D} / \mathrm{H}$ ratio at time $t, W$ is the remaining reservoir of water at time $t$, and $L$ is the amount of water lost from planet between time 0 and time $t$. If $\mathrm{D}$ and $\mathrm{H}$ escaped with equal ease, $F$ would be 1 and $X$ would be constant through time; if $H$ could escape but $D$ could not, then $F$ would be 0 . The $F$ value based on the most complete past model of Martian photochemistry is 0.32 , determined by eight kinetic processes modeled in detail [Yung et al., 1988]. Krasnopolsky et al. [1998] recently detected deuterium in the upper atmosphere of Mars by Hubble Space Telescope (HST) observations of Lyman $\alpha$ emission. They deduced $F=0.02 \pm 0.01$. (The error estimate for $F$ is ours, from theirs for $D / H$.) Any $F$ in this range challenges the photochemical model.

As discussed by Yung et al. [1988], water is the ultimate source of atmospheric $\mathrm{H}$, but molecular hydrogen $\left(\mathrm{H}_{2}\right)$ is the major carrier of hydrogen species to the upper atmosphere of Mars, where $\mathrm{H}$ escapes to space. Thus the $\mathrm{D} / \mathrm{H}$ ratio of molecular hydrogen can provide a basis for calculating $F$. The partitioning of $\mathrm{D}$ between HD and HDO in the atmosphere may be defined by:

$$
R=\left(\mathrm{HD} / \mathrm{H}_{2}\right) /\left(\mathrm{HDO} / \mathrm{H}_{2} \mathrm{O}\right)
$$

The relation between $F$ and $R$ is determined by the photochemical model, and $F$ is approximately proportional to $R$. To reconcile their measurements with the photochemical model, Krasnopolsky et al. [1998] suggested there might be a conceptual error in current photochemical models of Mars. They proposed that the $\mathrm{D} / \mathrm{H}$ ratio of escaping hydrogen could be determined by a thermodynamic equilibrium on the surface of Mars:

$$
\mathrm{HD}+\mathrm{H}_{2} \mathrm{O} \longleftrightarrow \mathrm{H}_{2}+\mathrm{HDO}
$$

According to kinetic theory, $R$ is 1.6 [Yung et al., 1988], but the equilibrium (3) implies $R=0.14$ at $200 \mathrm{~K}$, a temperature typical of the Martian surface. The measurements of deuterium with the HST imply $R=$ 
0.09 , much closer to the thermodynamic than the kinetic value. However, for the thermodynamics to prevail on Mars, the forward reaction of (3) must be sufficiently fast. Krasnopolsky et al. [1998] estimated the required rate constant $k_{3}$ as at least $10^{-23} \mathrm{~cm}^{3} \mathrm{~s}^{-1}$, not consistent with the laboratory rate constant of $10^{-33}$ $\mathrm{cm}^{3} \mathrm{~s}^{-1}$ [Lécluse and Robert, 1994]. There are two plausible resolutions of this paradox [Yung and Kass, 1998]. The first is that a reaction or fractionation hitherto neglected can reduce the value of $R$ in the photochemical model. The second is that a hitherto unknown catalyst on Mars can raise the rate coefficient of the forward reaction in (3) by ten orders of magnitude. In the laboratory experiments [Lécluse and Robert, 1994], the catalytic effects of charcoal, silica, phyllosilicates, and iron were found to be negligible.

The resolution of this puzzle is basic to our understanding the evolution of the Martian atmosphere. We could use the $F$ deduced from observations to model atmospheric evolution, but without knowing the fundamental processes determining $F$, we cannot trust extrapolations to the early atmosphere. For instance, if the current value of $F$ were influenced by catalysts on the Martian surface today, we would need to know whether the same catalysts were probable on early Mars.

We propose PHIFE as a simple and likely mechanism to resolve the discrepancy. Although UV photolysis is the primary destroyer of HDO in the Martian atmosphere, the UV cross sections of HDO have never before been measured. Existing measurements of $\mathrm{D}_{2} \mathrm{O}$ cross-sections [Laufer and McNesby, 1965] are coarse by modern standards. In modeling Mars, it has previously been assumed that HDO cross sections are the same as those of $\mathrm{H}_{2} \mathrm{O}$. Cross sections of HDO smaller than those of $\mathrm{H}_{2} \mathrm{O}$ would produce less $\mathrm{HD}$ relative to $\mathrm{H}_{2}$, implying a smaller $R$ in the model and better agreement with HST data.

\section{Measurements}

Laboratory measurements were carried out to determine the cross sections of $\mathrm{H}_{2} \mathrm{O}$ and its deuterium isotopomers. The light source was the synchrotron radiation dispersed with a 1-m Seya-Namioka monochromator located at the Synchrotron Radiation Research Center in Taiwan with a $1.5 \mathrm{GeV}$ storage ring [Tseng et al., 1995]. The monochromator was equipped with four gratings to cover the spectral range $30-300 \mathrm{~nm}$. We used the grating with 600 grooves/mm (blazed at 140 $\mathrm{nm}$ ) to cover the spectral range $100-300 \mathrm{~nm}$. The slit width was typically $0.05 \mathrm{~mm}$, corresponding to a spectral bandwidth of $\sim 0.1 \mathrm{~nm}$. The monochromator was scanned in either $0.1-\mathrm{nm}$ or $0.2-\mathrm{nm}$ steps with signalaveraging period of $3 \mathrm{~s}$ at each step. A small fraction of the light beam was reflected with a $\mathrm{CaF}_{2}$ plate and, after passing one additional $\mathrm{CaF}_{2}$ plate (thickness $2 \mathrm{~mm}$ ), irradiated onto a glass window coated with sodium salicylate. The fluorescence signal subsequently detected with a photomultiplier (Hamamatsu, R955) in a photon-counting mode was employed for normalization. The light transmitted from the $\mathrm{CaF}_{2}$ beamsplitter passed through an absorption cell equipped with two $\mathrm{CaF}_{2}$ end-windows, and irradiated onto a glass window coated with sodium salicylate. Fluorescence was detected similarly.

Two absorption cells were used, with inner diameter $39.5 \mathrm{~mm}$ and path lengths 10.2 and $113.3 \mathrm{~cm}$. To avoid variation in pressure due to irradiation or surface adsorption/desorption, a reservoir of volume $\sim 1382 \mathrm{~cm}^{3}$ was connected to the absorption cells. The absorption cell has a pressure port connected with two MKS Baratron pressure meters (model $127 \mathrm{AA}$, range 10 and 100 Torr). The spot size of the synchrotron radiation was $3.5 \times 1.0 \mathrm{~mm}$ at the entrance window of the absorption cell, and increased to $\sim 4.0 \times 1.5$ and $\sim 8.0 \times 2.5 \mathrm{~mm}$ at the terminal windows of the short and long absorption cells respectively.

At each wavelength, absorbance was plotted against number density and fitted to a line of least squares to yield the absorption cross section of the sample. Cross sections of HDO were determined using samples containing $\mathrm{H}_{2} \mathrm{O}$, $\mathrm{HDO}$, and $\mathrm{D}_{2} \mathrm{O}$, and assuming the absorption cross sections of pure $\mathrm{H}_{2} \mathrm{O}$ and $\mathrm{D}_{2} \mathrm{O}$ are as measured in this work. In addition to pure $\mathrm{H}_{2} \mathrm{O}$ and $\mathrm{D}_{2} \mathrm{O}$ samples, two mixtures were prepared from deionized $\mathrm{H}_{2} \mathrm{O}$ and pure $\mathrm{D}_{2} \mathrm{O}$ (Merck, Sharp and Dohme, isotopic purity $99.8 \%$ ) with molar ratios 1:2.81 and 1:4.02. The relative concentrations of $\mathrm{H}_{2} \mathrm{O}, \mathrm{D}_{2} \mathrm{O}$, and $\mathrm{HDO}$ in the gas sample mixtures were determined from the equilibrium constant $\mathrm{k}_{\text {eq }}=3.74$ [Pyper et al., 1967] and equilibrium vapor pressures [Kirshenbaum, 1951] of $\mathrm{H}_{2} \mathrm{O}$, $\mathrm{D}_{2} \mathrm{O}$, and $\mathrm{HDO}$ at $295 \mathrm{~K}$. The gaseous molar fractions of $\mathrm{H}_{2} \mathrm{O}, \mathrm{HDO}$, and $\mathrm{D}_{2} \mathrm{O}$ of these two samples were thus determined to be $(0.079,0.394,0.526)$ and $(0.046,0.328$, 0.625 ). For experiments with $\mathrm{D}_{2} \mathrm{O}$ or mixtures of the three isotopomers, at least 10 cycles of passivation were carried out. Absorbance of each sample was measured at 8 to 14 different pressures; the spectral range of measurements depends on pressure of the sample and the path length of the cell. Absorbance was calculated according to Beer's law. Data with absorbance greater than 2.0 were discarded to avoid saturation effects.

The resultant absorption cross sections of $\mathrm{H}_{2} \mathrm{O}, \mathrm{HDO}$, and $\mathrm{D}_{2} \mathrm{O}$ in the spectral range $140-195 \mathrm{~nm}$ are shown in Figure 1. (The data are available from website http://ams-bmc.srrc.gov.tw). A complete listing at 0.2$\mathrm{nm}$ intervals is available upon request. Variations in absorption cross sections of HDO determined from the two mixtures containing different fractions of HDO were typically within $5 \%$; averaged values are listed. Cross sections of $\mathrm{H}_{2} \mathrm{O}$ were compared with other recent measurements. Our $\mathrm{H}_{2} \mathrm{O}$ spectrum is nearly identical to that of Chan et al. [1993], who used low resolution dipole $\left(\mathrm{e}, \mathrm{e}^{\prime}\right)$ spectroscopy and TRK sum-rule normalization. In the spectral region $140-182 \mathrm{~nm}$, our $\mathrm{H}_{2} \mathrm{O}$ 


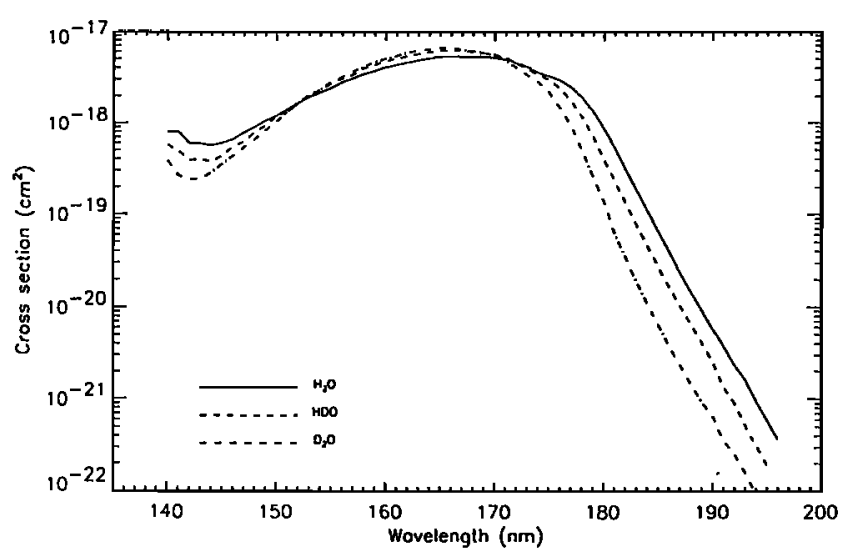

Figure 1. Laboratory measurements of the cross sections of $\mathrm{H}_{2} \mathrm{O}$ (solid line), $\mathrm{HDO}$ (dashed line), and $\mathrm{D}_{2} \mathrm{O}$ (dash dot line) from 140 to $195 \mathrm{~nm}$. The measurements were taken at $295 \mathrm{~K}$ with approximately $0.1 \mathrm{~nm}$ resolution.

results are also in agreement with those of Yoshino et al. [1996], who used a 12-cm cell and synchrotron radiation; there is a local maximum deviation of $7 \%$ near the peak of the band at $166 \mathrm{~nm}$. For wavelengths greater than $182 \mathrm{~nm}$, Yoshino et al. [1996] overestimated the cross sections. Because we used a much longer absorption cell, our measurements are expected to be more reliable in this spectral region; also, we extended the measurements to $195 \mathrm{~nm}$. Absorption cross sections of $\mathrm{D}_{2} \mathrm{O}$ determined in our work appear smaller in the valley (by $\sim 35 \%$ at $142.3 \mathrm{~nm}$ ) and greater at the peak (by $\sim 18 \%$ at $166.0 \mathrm{~nm}$ ) than those reported by Laufer and McNesby [1965]. Presumably this is because we used a smaller spectral width of the monochromator and carried out better passivation. $\mathrm{H}_{2} \mathrm{O}$ impurity increases absorbance in the valley and decreases absorbance at the peak, as shown in Figure 1. The absorption cross sections of $\mathrm{HDO}$ (and $\mathrm{D}_{2} \mathrm{O}$ ) are blue-shifted relative to those of $\mathrm{H}_{2} \mathrm{O}$. The theoretical reason is the difference in zero point energies, as first pointed out by Yung and Miller [1997] for PHIFE in $\mathrm{N}_{2} \mathrm{O}$ and verified by Rahn et al. [1998]. It is significant that the cross sections of the heavier isotopomers cross over around $170 \mathrm{~nm}$, as would be expected on the basis of the zero point energy theory.

\section{Application and Discussion}

The results from Figure 1 were used in a Mars model to evaluate the fractionating effect of the difference in photolytic cross sections of $\mathrm{H}_{2} \mathrm{O}$ and HDO. Figure 2 shows a comparison of photolysis rates $\left(\mathrm{cm}^{-3} \mathrm{~s}^{-1}\right)$ of $\mathrm{H}_{2} \mathrm{O}$ and $\mathrm{HDO}$ in the atmosphere of Mars. The $\mathrm{H}_{2} \mathrm{O}$ rate is taken from the model of Nair et al. [1994]. The HDO rate was computed with the same software, replacing the cross sections of $\mathrm{H}_{2} \mathrm{O}$ with those of HDO found in the present work. On a per-molecule basis, HDO photolysis in the upper atmosphere of Mars is about the same as that of $\mathrm{H}_{2} \mathrm{O}$. At these altitudes, most of the photons are absorbed near $170 \mathrm{~nm}$, where there is little difference between absorption by $\mathrm{HDO}$ and $\mathrm{H}_{2} \mathrm{O}$. But in the lower atmosphere, photolysis of HDO is 23 times less efficient than that of $\mathrm{H}_{2} \mathrm{O}$. In this region, shielding of UV radiation by $\mathrm{CO}_{2}$ becomes important. The photons that can penetrate to these levels are in the long-wave tail near $190 \mathrm{~nm}$, where the difference between $\mathrm{HDO}$ and $\mathrm{H}_{2} \mathrm{O}$ cross sections is large. An estimate using a revision of the model of Yung et al. [1988], taking into account of the correct average over a solar cycle [Pathare and Paige, 1997] brings the model value of the fractionation parameter $\mathrm{F}$ to 0.06 .

The past loss of water from Mars, based on extrapolation from present escape rates, was first estimated to be a few meters [Yung et al., 1988]. Subsequent revisions, taking account of sputtering induced by the solar wind, and of a more active early sun, raised the estimate to 50 $\mathrm{m}$ [Kass and Yung, 1995; Kass, 1999]. Using Eqn. (1) with $F$ in the range 0.02 to 0.06 , this would imply that the current water reservoir on Mars is equivalent to a global layer of 8-10 $\mathrm{m}$ [Kass and Yung, 1999], or about $10^{6} \mathrm{~km}^{3}$. This is consistent with the total ice volume of $1.2-1.7 \times 10^{6} \mathrm{~km}^{3}$ in the northern permanent polar cap estimated from data obtained by the Mars Orbiter Laser Altimeter [Zuber et al., 1998]. Reversing the argument, we may conclude that the existence of so much ice (assuming it is a pure water ice) in the polar cap today must imply, via Eqn. (1), that at least $50 \mathrm{~m}$ of water has escaped from Mars.

PHIFE is a process probably common in planetary atmospheres throughout the solar system, and may play an important part in reconstructing the evolution of the planets [Yung and DeMore, 1999]. There should be major differences, not yet measured, in the UV absorption cross sections for isotopomers of $\mathrm{HCl}, \mathrm{CH}_{4}$, and

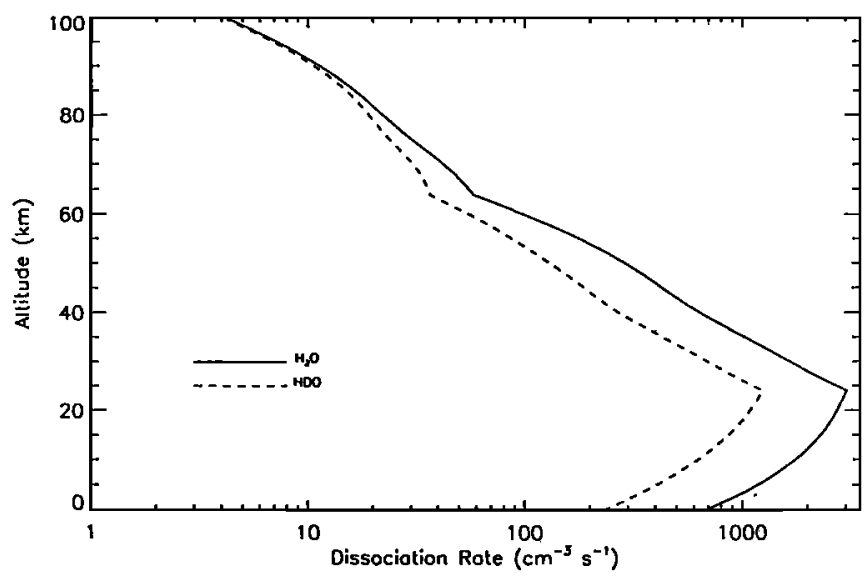

Figure 2. Comparison of photolysis rates $\left(\mathrm{cm}^{-3} \mathrm{~s}^{-1}\right)$ of $\mathrm{H}_{2} \mathrm{O}$ and and the relative rate of HDO in the atmosphere of Mars based on the model of Nair et al. The $\mathrm{H}_{2} \mathrm{O}$ rate is taken from the standard model. The HDO rate is computed by replacing the cross sections of $\mathrm{H}_{2} \mathrm{O}$ with those of HDO as measured in Figure 1. To obtain the actual photolysis of HDO on Mars, the HDO rate must be scaled by its mixing ratio relative to $\mathrm{H}_{2} \mathrm{O}$. 
$\mathrm{NH}_{3}$. These would imply differences in photodissociation rates for $\mathrm{HCl}$ and $\mathrm{DCl}$ on Venus, $\mathrm{CH}_{4}$ and $\mathrm{CH}_{3} \mathrm{D}$ on the giant planets and Titan, and $\mathrm{NH}_{3}$ and $\mathrm{NH}_{2} \mathrm{D}$ on the giant planets. For example, the stability of DCl relative to $\mathrm{HCl}$ might account for a long-standing puzzle on Venus, where D Lyman $\alpha$ has not been detected even though Venus is D-enriched by two orders of magnitude, relative to Earth [Clarke and Bertaux, 1989].

Acknowledgments. The authors thank $\mathrm{H}$. Nair for computing Figure 2, and D. M. Kass, C. M. Miller and B. P. Weiss for valuable comments. This research was supported in part by NASA grant NAG5-4022 and NSF grant AST9816409 to the California Institute of Technology.

\section{References}

Baker, V. R., M. H. Carr, V. C. Gulick, C. R. Williams, and M. S. Marley, Channels and valley networks, in Mars, H. H. Kieffer, B. M. Jakosky, C. W. Snyder, and M. S. Matthews, Eds. (The University of Arizona Press, Tucson, 1992).

Carr, M. H., Water on Mars (Oxford University Press, New York, 1996).

Chan, W. F., G. Cooper, and C. E. Brion, The electronic spectrum of water in the discrete and continuous regions: Absolute optical oscillator strengths for photoabsorption (6-200 eV), Chem. Phys., 178, 387-400, 1993.

Clarke, J. T. and J. L. Bertaux, Deuterium content of the Venus atmosphere, Nature, 398, 567-568, 1989.

Farquhar, J., M. H. Thiemens, and T. Jackson, Atmospheresurface interactions on Mars: $\Delta^{17} \mathrm{O}$ measurements from ALH84001, Science, 1580-1582, 1998.

Jakosky. B. M., Mars volatile evolution: implications of the recent measurement of ${ }^{17} \mathrm{O}$ in water from the SNC meteorites, Geophys. Res. Lett., 20, 1591-1594, 1993.

Kass, D. M., and Y. L. Yung, Water on Mars: Isotopic constraints on exchange between the atmosphere and the surface, submitted to Geophys. Res. Lett., 1999.

Kass, D. M., Change in the Martian atmosphere, Ph.D. thesis, California Institute of Technology, 1999.

Kass, D. M. and Y. L. Yung, Loss of atmosphere from Mars due to solar wind-induced sputtering, Science, 268, 697699, 1995.

Kass, D. M. and Y. L. Yung, Water on Mars: Isotopic constraints on exchange between the atmosphere and surface, Geophys. Res. Lett., this issue.

Kirshenbaum, I., Physical properties (Chapter 1) in Physical Properties and Analysis of Heavy Water, H. C. Urey and G. M. Murphy, Eds. (McGraw-Hill, New York, 1951).

Krasnopolsky, V. A., G. L. Bjoraker, M. J. Mumma, and D. E. Jennings, High-resolution spectroscopy of Mars at 3.7 and $8 \mu \mathrm{m}$ : A sensitive search for $\mathrm{H}_{2} \mathrm{O}_{2}, \mathrm{H}_{2} \mathrm{CO}, \mathrm{HCl}$, and $\mathrm{CH}_{4}$, and detection of HDO, J. Geophys. Res., 102, 6525-6534, 1997.

Krasnopolsky, V. A., M. J. Mumma, and G. R. Gladstone, Detection of atomic deuterium in the upper atmosphere of Mars, Science, 280, 1576-1580, 1998.

Laufer, A. H. and J. R. McNesby, Deuterium isotope effect in vacuum ultraviolet absorption coefficients of water and methane, Can. J. Chem., 43, 3487-3490, 1965.

Lécluse, C. and F. Robert, Hydrogen isotopic exchange reaction rates: Origin of water in the inner solar system, Geochim. Cosmoschim. Acta, 58, 2927-2939, 1994.

McElroy, M. B., Mars: an evolving atmosphere, Science, $175,443-445,1972$.
McElroy, M. B. and Y. L. Yung, Oxygen isotopes in the Martian atmosphere: implications for the evolution of volatiles, Planet. Space Sci., 24, 1107-1113, 1976.

Nair, H., M. Allen, A. D. Anbar, Y. L. Yung, and R. T. Clancy, A photochemical model of the Martian atmosphere, Icarus, 111, 124-150, 1994.

Owen, T., J. P. Maillard, C. deBergh, and B. L. Lutz, Deuterium on Mars: the abundance of $\mathrm{HDO}$ and the value of D/H, Science, 240, 1767-1770, 1988.

Pathare, A., and D. A. Paige, A reexamination of deuterium fractionation on Mars (abstract), Bull. Amer. Astron. Soc., 29, 977, 1997.

Pyper, J. W., R. S. Newbury, and G. W. Barton, Jr., Study of the isotopic disproportionation reaction between light and heavy water uing a pulsed molecular-beam mass spectrometer, J. Chem. Phys., 46, 2253-2257, 1967.

Rahn, T., H. Zhang, M. Wahlen, and G. A. Blake, Stable isotope fractionation during ultraviolet photolysis of $\mathrm{N}_{2} \mathrm{O}$, Geophys. Res. Lett., 25, 4489-4493, 1998.

Thiemens, M. H., T. L. Jackson, and C. A. M. Brenninkmeijer, Observation of a mass independent oxygen isotopic composition in terrestrial stratospheric $\mathrm{CO}_{2}$, the link to zone chemistry, and the possible occurrence in the Martian atmosphere, Geophys. Res. Lett., 22, 255-257, 1995.

Tseng, P. C., T. F. Hsieh, Y. F. Song, K. D. Lee, S. C. Chung, C. I. Chen, H. F. Lin, T. E. Dann, L. R. Huang, C. C. Chen, J. M. Chuang, K. L. Tsang, and C. N. Chang, Performance of the 1-m Seya-Namioka monochromator beam line at SRRC, Rev. Sci. Instrum., 66, 1815-1817, 1995.

Yoshino, K., J. R. Esmond, W. H. Parkinson, K. Ito, and T. Matsui, Absorption cross-section measurements of water vapor in the wavelength region $120 \mathrm{~nm}$ to $188 \mathrm{~nm}$, Chem. Phys., 211, 387-391, 1996.

Yung, Y. L. and W. D. DeMore, Photochemistry of Atmospheres (Oxford University Press, 1999).

Yung, Y. L. and D. M. Kass, Deuteronomy?: a puzzle of deuterium and oxygen on Mars, Science, 280, 1545-1546, 1998.

Yung, Y. L. and C. E. Miller, Isotopic fractionation of stratospheric nitrous oxide, Science 278, 1778-1780, 1997.

Yung, Y. L., J. S. Wen, J. P. Pinto, M. Allen, K. K. Pierce, and S. Paulson, HDO in the Martian atmosphere: implications for the abundance of crustal water, Icarus, 76, 146-159, 1988.

Zuber, M. T., D. E. Smith, S. C. Solomon, J. B. Abshire, R. S. Afzal, and 16 others, Observations of the north polar section of Mars from the Mars Orbiter Laser Altimeter, Science, 282, 2053-2060, 1998.

(Received May 27, 1999; revised July 20, 1999; accepted August 6, 1999.)

B. M. Cheng, E. P. Chew and C. P. Liu, Synchrotron Radiation Research Center, No. 1, R\&D Road VI, Hsinchu Science-Based Industrial Park, Hsinchu 30077, Taiwan, (bmchen@alpha1.srrc.gov.tw)

M. Bahou and Y. P. Lee, Department of Chemistry, National Tsing Hua University, 101, Sec. 2, Kuang Fu Road, Hsinchu 30043, Taiwan, (yplee@net.nthu.edu.tw)

Y. L. Yung and M. F. Gerstell, Division of Geological and Planetary Sciences, California Institute of Technology Mail Stop 150-21, Pasadena, CA 91125, (yly@gps.caltech.edu)

(Received May 27, 1999; revised July 20, 1999; accepted August 6, 1999.) 